\title{
Optimization of electrode shape for stripline beam position monitors
}

\author{
Yoshihiro Shobuda \\ JAEA, 2-4 Shirakata Shirane, Tokaimura, Nakagun, Ibaraki 319-1195, Japan \\ Yong Ho Chin \\ KEK, High Energy Accelerator Research Organization, 1-1 Oho, Tsukuba, Ibaraki 305-0801, Japan
}

(Received 14 January 2014; published 11 September 2014)

\begin{abstract}
The exponentially tapered beam position monitor has been proposed by Linnecar to produce a flat and wideband frequency response for beam position signals. However, it still has a large ringing fluctuation on the amplitude of the transfer function. This paper aims at improving the overall characteristics of the transfer function. With the help from the window function theory, we investigate the correlation between the shape of the electrode and the resulting transfer function. Finally, we propose a polynomial shape for the electrode that provides a much flatter response function. Three-dimensional results using the CST STUDIO are also presented to demonstrate the validity of the polynomial shape even in more realistic conditions.
\end{abstract}

DOI: 10.1103/PhysRevSTAB.17.092801

PACS numbers: 29.27.-a

\section{INTRODUCTION}

In the Japan Proton Accelerator Research Complex (J-PARC) [1] main ring (MR), transverse intrabunch oscillations have been observed in association with particle losses during the injection and at the onset of acceleration [2]. Recently, a new wideband (intrabunch) feedback system has been installed in the MR. It divides each bucket to 64 slices and acts on each slice as if it is a small bunch (bunchlet) in a narrow band feedback system.

Stripline position monitors, operated at $108.8 \mathrm{MHz}$, are used to pick up transverse signals from a beam. The same stripline position monitors are under consideration for monitoring electron cloud instabilities in the future, whose activities stretch to a rather high frequency, typically in the order of a few GHz. Therefore, the stripline position monitors are required to have a wideband and flat frequency response covering from $100 \mathrm{MHz}$ to a few $\mathrm{GHz}$.

The J-PARC stripline position monitor has four electrodes inside the vacuum chamber and they are connected to feedthroughs with resistors for the impedance matching. Based on the two-dimensional theory, the beam induced voltage at the resistors is proportional to the transfer function $F(\omega)$ [3] (In a simple case, the relation is derived in the Appendix). The transfer function $F(\omega)$ for a beam position monitor with electrodes of length $l$ is analytically given by [3]

$F(\omega)=i \omega \int_{0}^{\frac{2 l}{c}} \frac{1}{2} k\left(\frac{c t}{2}\right) e^{-i \omega t} d t=\frac{i \omega}{c} \int_{0}^{l} k(z) e^{-i \frac{2 \omega}{c} z} d z$

Published by the American Physical Society under the terms of the Creative Commons Attribution 3.0 License. Further distribution of this work must maintain attribution to the author(s) and the published article's title, journal citation, and DOI. where $i$ is the imaginary unit, $\omega=2 \pi f$ is the angular frequency, $k(z)$ is the coupling function to the fields at point $z$ with the electrode, and the velocity of the beam is approximated by the speed of light $c$. Here, it is assumed that the height of the electrodes from the chamber surface, their width and thicknesses are varied proportionally to $k(z)$ along the longitudinal direction for the impedance matching.

When the electrodes have a conventional rectangular shape $[k(z)=$ constant $]$, the transfer function has notches in the amplitude, as shown by the red line in Fig. 1. In order to improve the transfer function to a flatter shape, Linnecar has proposed the electrode shape whose width and height from the chamber surface decrease exponentially in the longitudinal direction [4]. The improved transfer function is shown by the blue line in Fig. 1. Beam-based studies have been done to demonstrate the advantage of Linnecarshape electrodes in real machines [5]. However, the transfer function of the exponential coupler has still a large fluctuation in its amplitude.

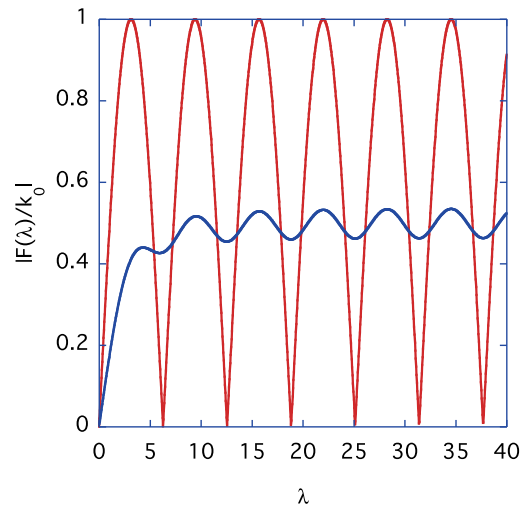

FIG. 1. The absolute value of transfer function for the coupling $k(z)=k_{0}$ (red) and that for $k(z)=k_{\text {linnecar }}(z)$ (blue) where $a=2.63$ is chosen. 
As seen in Eq. (1), the transfer function is defined by the Fourier transform of the coupling $k(z)$ on the confined region $(0<z<l)$. In other words, the function $k(z)$ plays a window function for the Fourier transform of the step function that takes nonzero value only in the region $(0<z<l)$. This identification of $k(z)$ suggests that the theory of the window function may provide us good guidelines to find a suitable function for $k(z)$ in the optimization of the transfer function.

In the next section, we will investigate the correlations between the transfer function and the coupling $k(z)$.

\section{CORRELATIONS BETWEEN THE TRANSFER FUNCTION AND THE SHAPE OF ELECTRODE}

When the coupling $k(z)$ is constant $\left(k=k_{0}\right)$, the transfer function is given by

$$
\begin{aligned}
F_{\text {nowin }}(\lambda) & =\frac{k_{0}}{2}\left(1-e^{-i \lambda}\right), \\
\lambda & =\frac{2 \omega l}{c} .
\end{aligned}
$$

Here the dimensionless parameter $\lambda$ is introduced. The absolute value of the transfer function is

$$
\left|F_{\text {nowin }}[\lambda(\omega)]\right|=k_{0}\left|\sin \frac{\lambda}{2}\right|=k_{0}\left|\sin \frac{\omega l}{c}\right|,
$$

which is shown by the red line in Fig. 1. The result has notches in every period of $\Delta f=c / 2 l$. It behaves as a half sin, and repeats indefinitely in frequency $f$.

Linnecar has proposed the exponentially decaying function for $k(z)$ :

$$
k_{\text {linnecar }}(z)=k_{0} e^{-\frac{a z}{l}}
$$

where $a$ is a positive dimensionless parameter, defining the degree of the exponential tapering of the electrode. When we determine the parameter $a$, the important point is that the absolute value of the transfer function should quickly rise along $\lambda$ as well as the fluctuation of it should be significantly suppressed. From this point, the parameter $a$ is chosen to be 2.63 in this paper. Finally, the direct comparison between this result and the authors' suggestion will be done.

By substituting Eq. (5) into Eq. (1), the transfer function is given by

$$
F_{\text {linnecar }}(\lambda)=\frac{k_{0}}{2}\left[\frac{i \lambda\left(1-e^{-a-i \lambda}\right)}{(a+i \lambda)}\right] .
$$

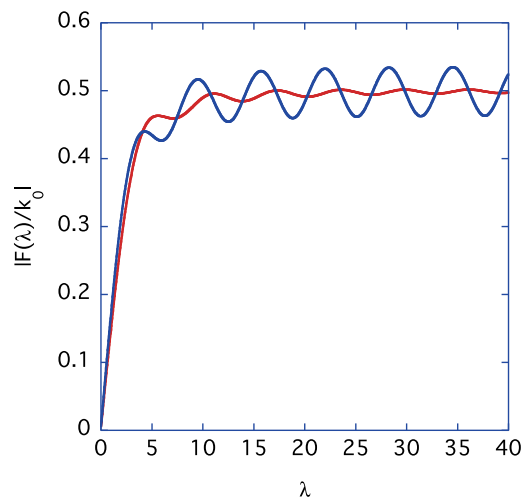

FIG. 2. The absolute value of the transfer function for the coupling $k_{\text {linnecar }}^{\text {new }}(z)$ (red) and that for $k_{\text {linnecar }}(z)$ (blue).

The blue line in Fig. 1 shows the calculation results. This transfer function has no notch, but still has the large fluctuating amplitude.

The theory of the window function indicates that the fluctuation mainly comes from the truncation of the exponential shape at the end of the electrode [6]. To make the end value of $k(z)$ zero, let us define a new coupling function $k_{\text {linnecar }}^{\text {new }}(z)$ by subtracting the constant values of $k_{\text {linnecar }}(l)$ from $k_{\text {linnecar }}(z)$, as

$$
k_{\text {linnecar }}^{\text {new }}(z)=k_{0}\left(\frac{e^{-\frac{a z}{l}}-e^{-a}}{1-e^{-a}}\right) .
$$

The corresponding transfer function is given by

$$
F_{\text {linnecar }}^{\text {new }}(\lambda)=\frac{k_{0}}{2}\left[\frac{a\left(1-e^{-i \lambda}\right)+i\left(1-e^{a}\right) \lambda}{\left(1-e^{a}\right)(a+i \lambda)}\right] .
$$

Note that Eq. (8) converges to $k_{0} / 2$ as $\omega$ goes to infinity.

Figure 2 shows numerical comparison between $F_{\text {linnecar }}^{\text {new }}(\lambda)$ and $F_{\text {linnecar }}(\lambda)$. The red and the blue lines show $\left|F_{\text {linnecar }}^{\text {new }}(\lambda) / k_{0}\right|$ and $\left|F_{\text {linnecar }}(\lambda) / k_{0}\right|$, respectively. As expected, the fluctuation is drastically reduced.

The remaining ringing effect in the red line is considered to be due to the nonzero derivative of $k(z)$ at $z=l$. To see how the ringing effect can be improved by making the derivative of $k(z)$ at $z=l$ zero, let us try the BlackmanHarris window function for $k(z)$ [6]:

$$
\begin{aligned}
k_{\text {blackman-harris }}(z)= & k_{0}\left\{0.35875-0.48829 \cos \left[\pi\left(\frac{z}{l}-1\right)\right]\right. \\
& +0.14128 \cos \left[2 \pi\left(\frac{z}{l}-1\right)\right] \\
& \left.-0.01168 \cos \left[3 \pi\left(\frac{z}{l}-1\right)\right]\right\}
\end{aligned}
$$

The left part of Fig. 3 shows the shapes of the electrodes where the half width of the electrode at $z=0$ and the length 

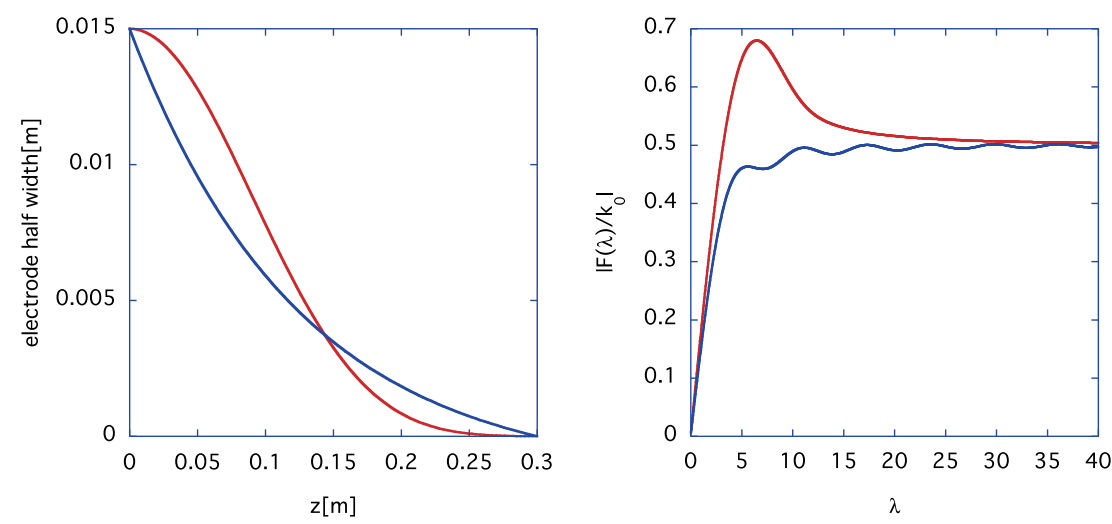

FIG. 3. The right part shows the absolute value of the transfer function for the coupling $k_{\text {linnecar }}^{\text {new }}(z)$ (blue) and that for $k_{\text {blackman-harris }}(z)$ (red). The corresponding shapes of the electrodes are shown in the left part by the lines with the same colors. The half width of electrode at $z=0$ and the length $l$ are assumed to be $15 \mathrm{~mm}$ and $0.3 \mathrm{~m}$, respectively.

$l$ are assumed to be $15 \mathrm{~mm}$ and $0.3 \mathrm{~m}$, respectively. The blue and the red lines show the shapes $k_{\text {linnecar }}^{\text {new }}(z)$ and $k_{\text {blackman-harris }}(z)$, respectively. The lines with the same colors in the right part of Fig. 3 show the corresponding transfer functions. In the Blackman-Harris window (the red line), the fluctuation in the amplitude practically vanishes at high frequency. On the other hand, a large overshoot occurs at low frequency. Comparison of the blue line with the red one in the left part suggests that the overshoot is caused by the convex surface toward upward around $z=0$ (the red line).

To test this conjecture, let us consider a simple polynomial shape for the coupling $k(z)$ :

$$
k(z)=k_{0} \frac{(l-z)^{\sigma}}{l^{\sigma}}
$$

where $\sigma \geq 1$, and let us try to minimize the fluctuation of the absolute value of the transfer function $F^{\sigma}(\lambda)$ by optimizing the parameter $\sigma$. The transfer function $F^{\sigma}(\lambda)$ is given by

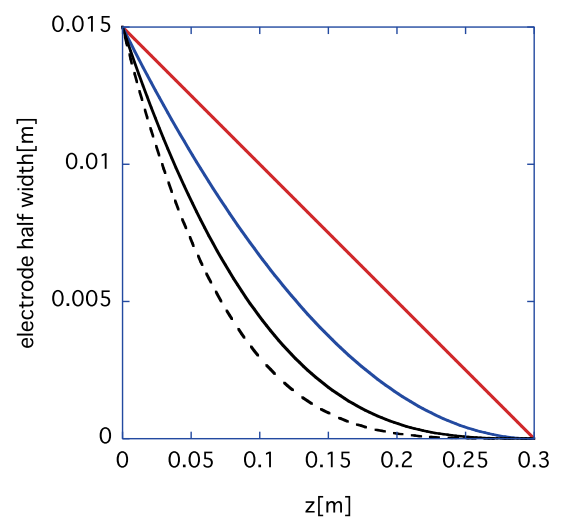

$$
F^{\sigma}(\lambda)=-\frac{k_{0}}{2} \frac{e^{-i \lambda}(\Gamma[1+\sigma]-\Gamma[1+\sigma,-i \lambda])}{(-i \lambda)^{\sigma}}
$$

where $\Gamma(z)$ and $\Gamma(a, z)$ are the $\Gamma$ and the incomplete $\Gamma$ functions, respectively [7].

Let us numerically compare $\left|F^{\sigma}(\lambda) / k_{0}\right|$ for various values of $\sigma$. The results for $\sigma=1,2,3,4$ are shown in the right part of Fig. 4 by the red solid, the blue solid, the black solid and the black dashed lines, respectively. The left part shows the shapes of electrodes where the half width of the electrode at $z=0$ and the length $l$ are assumed to be $15 \mathrm{~mm}$ and $0.3 \mathrm{~m}$, respectively. The absolute values of $F^{\sigma}(\lambda)$ fluctuate more for smaller values of $\sigma$, because of the smaller negative curvatures at the origin $z=0$. The flatness of the amplitude at high frequency is improved for a larger $\sigma$. We can see this characteristic by expanding Eq. (11) for large $\lambda$. The resulting approximate formula,

$$
F^{\sigma}(\lambda) \sim \frac{k_{0}}{2}\left(1-\frac{e^{i\left(\frac{\pi \sigma}{2}-\lambda\right)} \Gamma[1+\sigma]}{\lambda^{\sigma}}\right)
$$

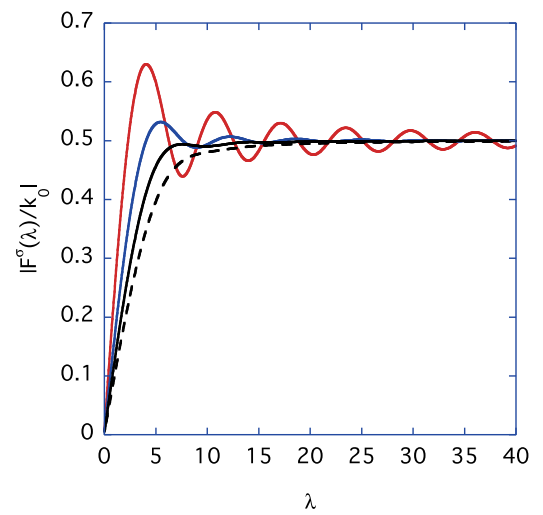

FIG. 4. The absolute values of transfer function $\left|F^{\sigma}(\lambda) / k_{0}\right|$ (right) and the shapes of electrode (left) for various values of $\sigma$. The red solid and the blue solid and the black solid and the black dashed lines depict the results of $\sigma=1,2,3$ and 4, respectively. The half width of the electrode at $z=0$ and the length $l$ are assumed to be $15 \mathrm{~mm}$ and $0.3 \mathrm{~m}$, respectively. 


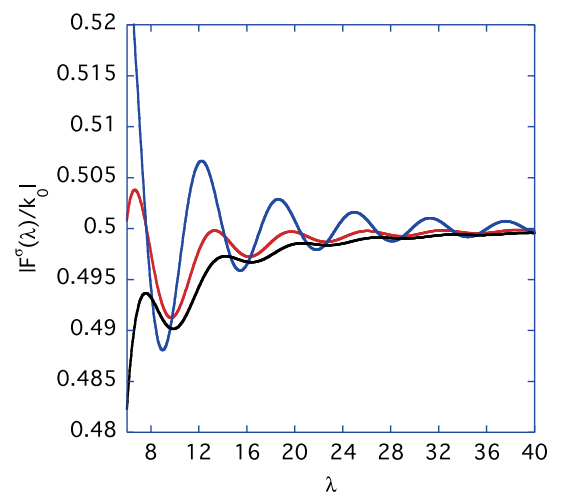

FIG. 5. The $\sigma$ dependence of $\left|F^{\sigma}(\lambda) / k_{0}\right|$ is shown. The results for the values of $\sigma$ are 2 (blue), 2.63 (red) and 3 (black) are shown.

approaches to $k_{0} / 2$ at high $\lambda$. It converges faster when $\sigma$ is larger.

However, the transfer function rises slowly as a function of $\lambda$, as $\sigma$ becomes larger. This behavior is understood by expanding Eq. (11) for small $\lambda$ as

$$
F^{\sigma}(\lambda) \sim \frac{k_{0}}{2} \frac{i \lambda}{(1+\sigma)}
$$

and by identifying its absolute value to $k_{0} / 2$ :

$$
\frac{k_{0}}{2} \frac{\lambda}{(1+\sigma)}=\frac{k_{0}}{2}
$$

The value of $\lambda$ (or, the frequency $f$ ) where the transfer function reaches the flat region is given by

$$
\lambda_{s}\left(\equiv \frac{4 \pi l f_{s}}{v}\right) \simeq 1+\sigma .
$$

It increases linearly as a function of $\sigma$.

Let us define the optimization procedure of $\sigma$ in the following way. First, we set the region of $\lambda, \lambda_{\text {low }}<\lambda<\lambda_{\text {up }}$, where the optimization of $\left|F^{\sigma}(\lambda)\right|$ is performed. In this region, we try to minimize the function $I(\sigma)$ defined by

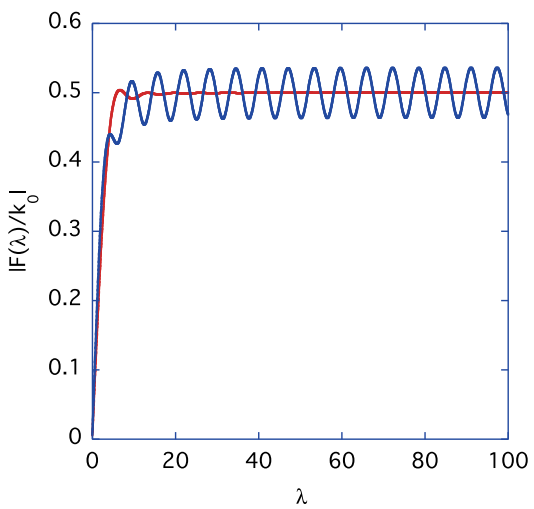

$$
I(\sigma)=\int_{\lambda_{\mathrm{low}}}^{\lambda_{\mathrm{up}}} d \lambda\left(\left|\frac{F^{\sigma}(\lambda)}{k_{0}}\right|-0.5\right)^{2} .
$$

For $\lambda_{\text {low }}=6$ and $\lambda_{\text {up }}=40$, the optimized $\sigma$ is 2.63 [the choice of $\lambda_{\text {up }}$ is not important, because $F^{\sigma}(\lambda)$ converges to $k_{0} / 2$ for large $\lambda$ ]. Figure 5 shows the cases of $\sigma=2,2.63$ and 3, by the blue and the red and the black lines, respectively. The oscillating amplitude is minimized at $\sigma=2.63$, and it is almost symmetrical about the $\left|F^{\sigma}(\lambda) / k_{0}\right|=0.5$ line.

Once the parameter $\sigma$ is determined, the transfer functions depend only on the parameter $\lambda$ : it is basically the product of the electrode length $l$ and the frequency $f$. Now, the only way to increase the rising slope of $\left|F^{\sigma}(\lambda)\right|$ as a function of frequency $f$ is to increase the length of electrode $l$. The rising slope is proportional to the inverse of $l$. For the value of $\sigma=2.63$, the frequency $f_{s}$ (at which, the transfer function reaches the flat region) is approximated as

$$
f_{s}[\mathrm{~Hz}] \simeq \frac{10^{8}}{l[\mathrm{~m}]}
$$

Simultaneously, the region where the fluctuation in the amplitude occurs contracts toward the lower frequency, as $l$ is longer. Thus, the longer the length of the electrode, the better the characteristic of the transfer function.

Finally, let us compare the amplitude and the phase of $F_{\text {linnecar }}(\lambda)$ for $a=2.63$ and those of $F^{\sigma}(\lambda)$ for $\sigma=2.63$. The results are shown in Fig. 6 by the blue and the red lines, respectively. The oscillational behaviors of $F_{\text {linnecar }}(\lambda)$ almost disappear in $F^{\sigma}(\lambda)$. The reason why the same value of 2.63 is chosen for the parameters $a$ and $\sigma$ is that $-\sigma k_{0} / l$ and $-a k_{0} / l$ are identical to the derivative of the coupling $k^{\prime}(z)$ at $z=0$. The fluctuation of the amplitude can be reduced by increasing the parameter $a$. However, it decreases the rising slope of $\left|F_{\text {linnecar }}(\lambda)\right|$ at the same time. In conclusion, Fig. 6 demonstrates a remarkable improvement of the flatness in the transfer function by the proposed polynomial shape of the electrode.

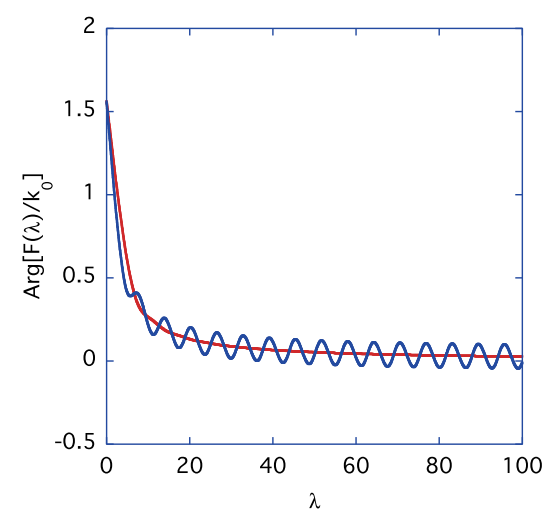

FIG. 6. The phase (right) and amplitude (left) of the transfer function for different shapes of electrodes. The red and the blue lines show the results for the shape given by Eq. (10) with $\sigma=2.63$ and those for Eq. (5) with $a=2.63$, respectively. 


\section{THREE-DIMENSIONAL SIMULATION RESULTS}

In the previous section, we have optimized the shape of the electrode based on the two-dimensional theory. In reality, however, the height of the electrodes from the chamber surface, and their width and thicknesses should be varied along the position $z$ in order to preserve characteristic impedance between the electrode and the chamber. Moreover, it is important to satisfy the impedance matching condition at both ends of the electrodes. For the impedance matching, the sharp tip of the electrode needs to have finite width, despite that it should have zero width for the ideal polynomial shape given by Eq. (10). In this section, we show some three-dimensional simulation results in more realistic configuration to demonstrate that the proposed polynomial shape indeed improves the characteristic of the transfer function.

The simulation study is done by using the threedimensional code CST STUDIO [8]. The code CST STUDIO SUITE 2014 SP3 implements WAKE SOLVER to calculate the excited electromagnetic fields, when a beam passes through the chamber embedding electrodes. For the shape of the electrodes, three kinds of shapes are considered: the rectangular shape, the exponential shape with $a=2.63$ and the polynomial shape with $\sigma=2.63$. Due to practical reason of the code to maintain sufficiently large mesh sizes in space, the electrodes are truncated at $190 \mathrm{~mm}$ length. In the simulations, cares are taken so that the characteristic impedance of $50 \Omega$ is preserved along the position $z$. Both ends of the electrodes are terminated with matched resistors. The inner and the outer radii of the chamber are 65 and $70 \mathrm{~mm}$, respectively, to simulate the chamber typically used at MR in J-PARC [1]. The rms bunch length used is $10 \mathrm{~mm}$. The parameter "simulated wakelength" in WAKE SOLVER is $1 \mathrm{~m}$.

The transfer function is proportional to the Fourier transform of the beam-induced voltages on the upstream side port (the wider end of the electrode) among the two, divided by the Fourier transform of the shape of the beam current. Figure 7 shows a schematic picture of three types of electrodes. Both ends of the electrodes are terminated with
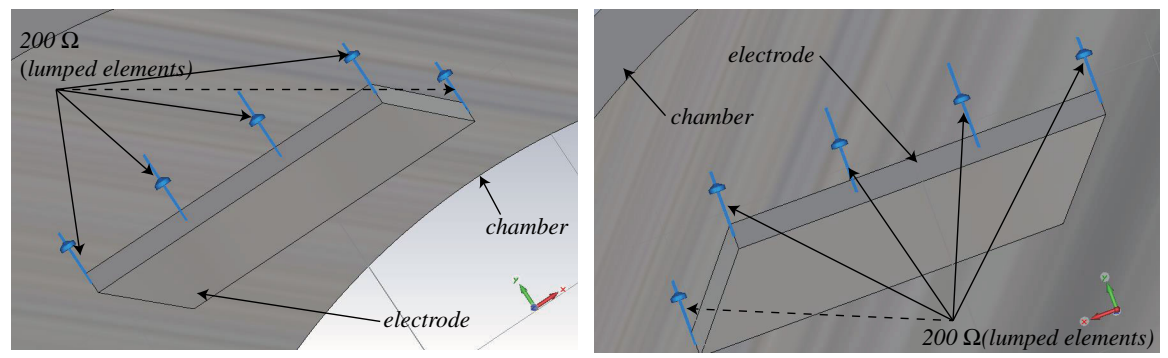

(a)
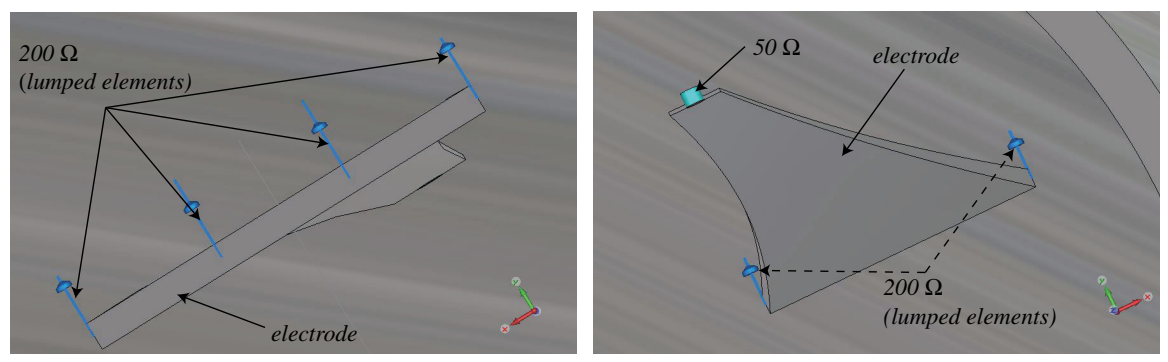

(b)
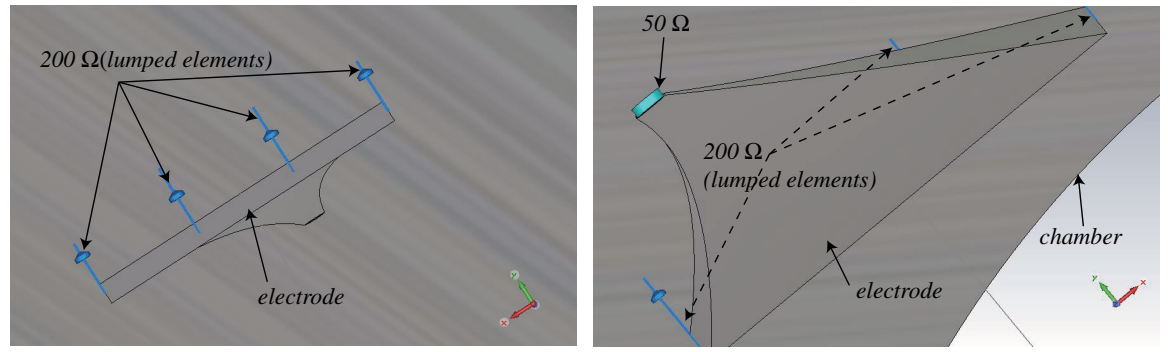

(c)

FIG. 7. A schematic picture of the electrode, where the ends are terminated with resistors in the vacuum chamber. (a) The rectangular shape, where the left and the right parts show the upstream and the downstream sides, respectively. (b) The exponential shape, where the left and the right parts show the upstream (wider) and the downstream (narrower) ends, respectively. (c) The polynomial shape, where the left and the right parts show the upstream (wider) and the downstream (narrower) ends, respectively. 


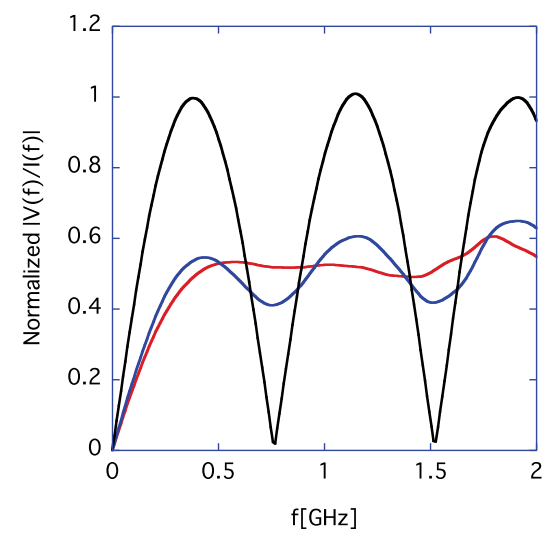

FIG. 8. The black, the blue and the red lines show the results for the electrode shapes; the rectangular one, the exponential one, and the polynomial one, respectively, where the ends are terminated with resistors in the vacuum chamber. All results are divided by the peak value at $0.38 \mathrm{GHz}$ for the rectangular shape. the matched resistors inside the chamber. The wider width is terminated with four $200 \Omega$ resistors in parallel, which are modeled by using the "lumped elements" implemented in CST STUDIO. The reason why this modeling is adopted is that this provides the beam-induced voltage at the resistor by checking "Monitor voltage and current" option in the "lumped elements." The resistor at the downstream side of the electrode, except in the case of the rectangular shape, is made of a cylindrical resistive material with the radius $1 \mathrm{~mm}$. The absolute values of the simulated transfer functions are shown in Fig. 8. For the rectangular shape shown by the black line, several sharp dips appear as expected. Comparison between the blue line for the exponential shape and the red one for the polynomial shape demonstrates that the oscillational behavior of the transfer function is remarkably suppressed below $1.5 \mathrm{GHz}$ for the polynomial shape, compared to the exponential one.

However, in reality, the induced voltage needs to be picked up outside the chamber through a feedthrough, not
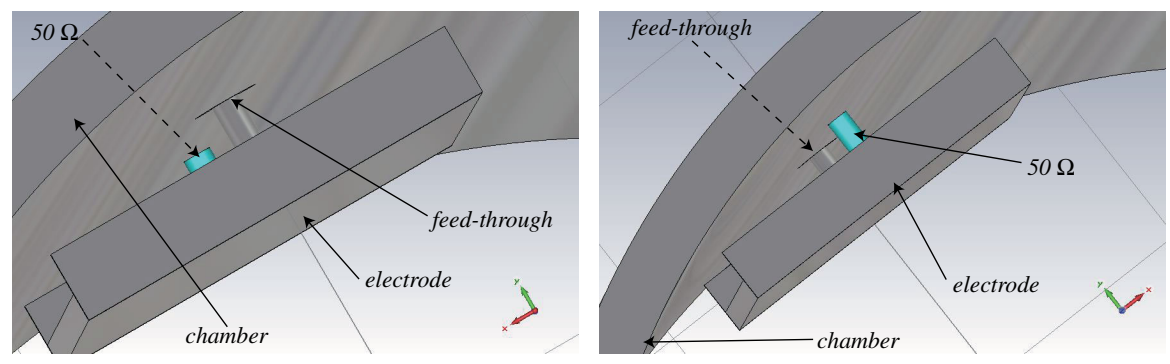

(a)
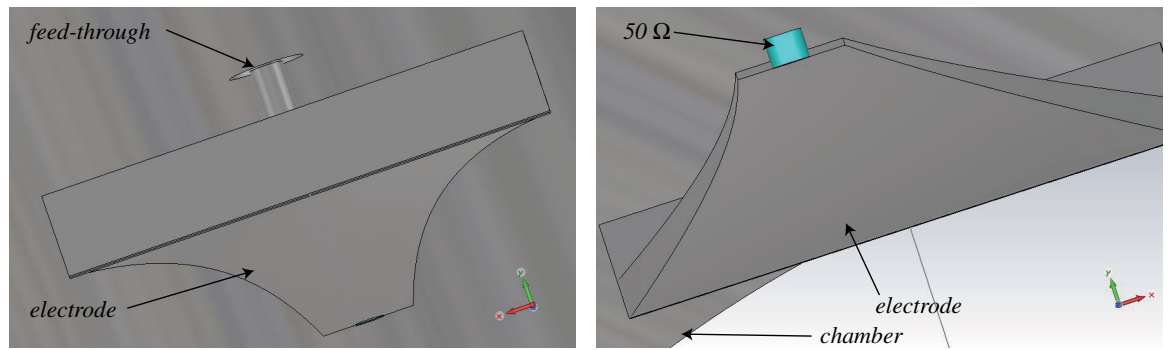

(b)
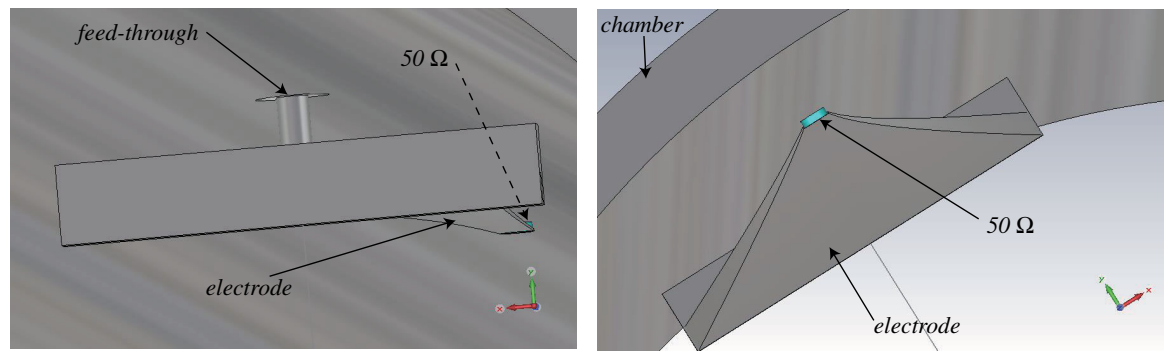

(c)

FIG. 9. A schematic picture of the electrode, where one end (upstream side) of the electrode is terminated with a $50 \Omega$ resistor (lumped elements) behind the feedthrough. The resistor is hidden in all these figures. (a) The rectangular shape, where the left and the right parts show the upstream and the downstream sides, respectively. (b) The exponential shape, where the left and the right parts show the upstream (wider) and the downstream (narrower) ends, respectively. (c) The polynomial shape, where the left and the right parts show the upstream (wider) and the downstream (narrower) ends, respectively. 


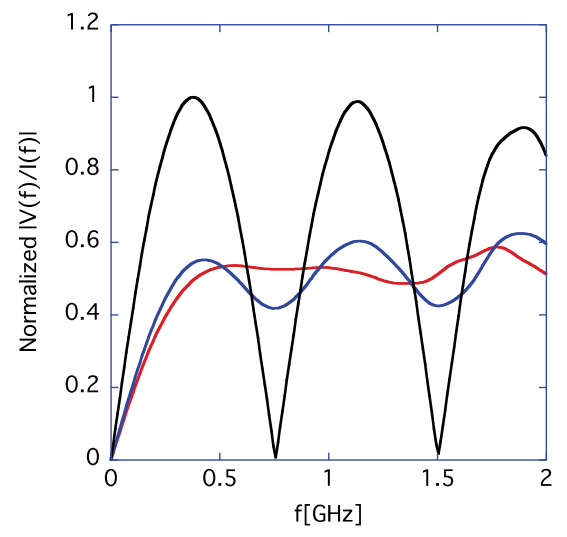

FIG. 10. The black, the blue and the red lines show results for the electrode shapes; the rectangular one, the exponential one, and the polynomial one, respectively, where one end (upstream side) of the electrode is terminated with a $50 \Omega$ resistor (lumped elements) behind the feedthrough. All results are divided by the peak value at $0.38 \mathrm{GHz}$ for the rectangular shape.

inside the chamber. The feedthrough is modeled by a coaxial structure with the inner radius $1 \mathrm{~mm}$ and the outer radius $2.3 \mathrm{~mm}$. Figure 9 shows a more realistic simulation with the feedthrough installed. One side of the feedthrough is terminated with a $50 \Omega$ resistor (lumped elements) and the other side is connected to the end of the electrode by bending the electrode perpendicularly at the end. The results are shown in Fig. 10. The black, the blue and the red lines show the results for the electrode shapes; the rectangular one, the exponential one, and the polynomial one, respectively. The frequency characteristics of these results are not considerably changed, compared to those of the previous results shown in Fig. 8. One can still identify the significant improvement of the transfer function by the polynomial shape. Figures 8 and 10 indicate the importance of impedance matching at the ends of the electrodes in order to take advantage of the optimized shape of the electrodes.

\section{SUMMARY}

This study is aimed at eliminating the ringing fluctuation of the transfer function in the exponential electrode. Applying the Blackman-Harris window function to the electrode shape suggests that the smoothly tapered shape toward zero at its end is the key to reduce the ringing effect, but the zero slope at the beginning causes the overshoot of the transfer function at low frequency. From these results, we propose the polynomial shape of electrode that satisfies both conditions: the smooth tapering to zero at the end and the negative slope at the beginning.

The polynomial shape of the electrode is parametrized by the dimensionless parameter $\sigma$. When $\sigma$ is large, the flatness of the transfer function is improved, but its rising slope becomes smaller. The optimized value of $\sigma$ is found to be about 2.6, regardless of the electrode length $l$. Practically speaking, the rising slope of the transfer function can be still improved by making the electrode longer. The longer electrode also reduces the remaining ringing effect at low frequency. In these regards, a longer electrode is preferable for the two effects: a flatter transfer function and its faster rising slope at low frequency.

These findings are based on the two-dimensional theory. There, it is implicitly assumed that the characteristic impedance of the electrodes is preserved along the beam chamber and special cares are taken for the impedance matching at the ends of the electrodes. The electrode needs to be tilted down toward the tip, proportionally to its width and thickness. The upstream port of the electrode is connected to a feedthrough by bending the electrode perpendicularly at the end, and is terminated with a $50 \Omega$ resistor outside of the vacuum vessel to absorb the comoving charges. The downstream tip of the electrode needs a finite size for the impedance matching. Nevertheless, the three-dimensional simulation study demonstrates that the polynomial electrode with all these attachments and cares still has a more significant flatter transfer function than the exponential one.

In the future we are planning to benchmark our calculations with real measurements.

\section{ACKNOWLEDGMENTS}

The authors would like to thank K. Nakamura, K. Takata, M. Tobiyama and T. Toyama for discussions. The authors also would like to thank all members of the J-PARC project at JAEA/KEK.

\section{APPENDIX: RELATION BETWEEN THE BEAM- INDUCED VOLTAGE ON ONE ELECTRODE AND THE TRANSFER FUNCTION $\boldsymbol{F}(\boldsymbol{\omega})$}

Let us assume an electrode with the length $l$, the inductance $L$ and the capacitance $C$ in a vacuum chamber. Both ends of the electrode are terminated with resistors $R$. Based on the transmission line model, the voltage $\tilde{V}$ and current $\tilde{I}$ at the position $z$ on the electrode, excited by a beam current $I_{0}$ with its frequency $f$, follow

$$
\begin{gathered}
\frac{d \tilde{V}(z)}{d z}=-i \omega L(z) \tilde{I}(z)-i \omega M(z) I_{0} e^{-i \frac{i_{c}}{c}}, \\
\frac{d \tilde{I}(z)}{d z}=-i \omega C(z) \tilde{V}(z),
\end{gathered}
$$

where $\omega=2 \pi f$ and $M(z)$ is the mutual inductance between the beam and the electrode at $z$. If the characteristic impedance $\sqrt{L / C}$ does not depend on $z$ and it is identical to $R$, the induced voltage at $z=0$ is given by

$$
\tilde{V}(z=0)=\frac{i \omega I_{0}}{2} \int_{0}^{l} d z^{\prime} M\left(z^{\prime}\right) e^{-i 2 \frac{\omega}{c} z^{\prime}}
$$

which is basically identical to the transfer function $F(\omega)$ in Eq. (1). 
Following Oliver [3], the sign of the imaginary unit $i$ is chosen in order that the corresponding function $v(t)$ in time domain is given by

$$
v(t)=\int_{-\infty}^{\infty} \frac{d \omega}{2 \pi} \tilde{V} e^{i \omega t}
$$

[1] http://j-parc.jp/index-e.html.

[2] K. Nakamura, T. Toyama, M. Okada, M. Tobiyama, Y. H. Chin, T. Obina, T. Koseki, and Y. Shobuda, Intra-bunch Feedback System for the J-PARC Main Ring in Proceedings of IPAC14, Dresden, Germany (JACoW, 2014), THOAA03.
[3] B. M. Oliver, Proc. IRE 42, 1686 (1954).

[4] T. P. R. Linnecar, Report No. CERN-SPS-ARF-SPS/78/17, 1978; T.P. R. Linnecar, in Proceedings of the 1979 Particle Accelerator Conference, San Francisco (Institute of Electrical and Electronics Engineers, New York, 1979) [IEEE Trans. Nucl. Sci. 26, 3409 (1979)].

[5] R. de Maria, C. Boccard, W. Höfle, G. Kotzian, C. Montava, and B. Salvant, in Proceedings of the International Particle Accelerator Conference, Kyoto, Japan (ICR, Kyoto, 2010), WEPEB054.

[6] F. G. Harris, Proc. IEEE 66, 51 (1978).

[7] M. Abramowitz and I. Stegun, Handbook of Mathematical Functions-With Formulas, Graphs, and Mathematical Tables (Dover, New York, 1974).

[8] CST STUDIO SUITE, https://www.cst.com/. 